\title{
Should all choices count? Using the cut-offs approach to edit responses in a choice experiment.
}

\begin{abstract}
$\underline{\text { Abstract }}$
Should we give equal weight to all responses in a choice experiment? Previous Choice Modelling papers have considered the issue of the internal consistency of choices - such as the extent to which strictly-dominated options are chosen. In this paper, a different focus is employed, namely the extent to which people choose options which violate their stated upper or lower limits for the acceptable levels for individual attributes. Since hypothetical overstatement of WTP has been a focus in stated preference studies, we concentrate on violations of stated upper limits of WTP, and explore the effects of "editing" such choices using a variable censoring rule and alternative approaches to re-classifying choices. The empirical case study is a choice experiment on eco-tourists in Rwanda. Our main conclusion is that the suggested approach offers a useful way of imposing consistency on choices, and that editing choices in this manner has an appreciable impact on estimated willingness to pay. However, issues remain with regard to explaining why people apparently violate their stated maximum willingness to pay.
\end{abstract}

Keywords: choice modelling, choice consistency, conservation, cut-offs, eco-tourism, random parameters logit,

Second Revised version, April 2009. 


\section{Introduction}

Choice Modelling papers have considered the issue of the internal consistency of choices - such as the extent to which strictly-dominated options are chosen, or whether respondents consistently choose the same alternative when a choice set is repeated as part of a survey (Hanley, Wright and Koop, 2002). Inconsistent responses can then be removed from the data set before a choice model is estimated. There is also a wider literature on the determinants of choice consistency, which considers issues such as the role of choice complexity and the use of heuristics (de Shazo and Fermo, 2002; Amir and Levev, 2008). In this paper, a different focus is employed, namely the extent to which people choose options which violate their stated upper or lower limits for the "acceptable" levels for individual attributes. Swait (2001) proposed that how people choose between alternatives might be represented using a "cut-offs" approach, whereby respondents specify minimum or maximum acceptable levels for different attributes. Choice sets which violate these levels can be either rejected (in the "hard" cut-offs version of the model), or else attract a utility penalty in the "soft" cut-offs version. The soft cut-offs alternative, described in detail below, allows for potential kinks in the utility function to be included. Here, we extend the method to allow for choices which violate an individually-stated maximum Willingness to Pay to be re-classified, and show that this produces substantial effects on parameter estimates and on willingness to pay for changes in attribute levels.

Indeed, hypothetical over-statement of WTP has been a focus of critical review in stated preference studies, leading to the notion that WTP estimates from choice experiments are in some senses "too high" (Carlsson and Martinsson, 2001; Harrison and Rustrom, 2005; List et al, 2006). For this reason, we concentrate here on violations of stated upper limits for the price attribute : that is, instances where individuals choose options which are more expensive than the maximum they indicate they are willing to pay. We explore the effects of 
editing such choices using a variable censoring rule, and with alternative approaches to reclassifying choices. People who choose options which are in excess of the most they say they would pay for the good might be viewed as not considering the price attribute carefully enough (Campbell, 2008), behaviour which can be tested for in a number of ways. Here, we take the position that individuals would not actually be willing to buy the good if they chose options which imply a violation of their stated maximum WTP by a certain percentage. This leads us to re-classify such choices as "no purchase" (we also explore the effects of simply deleting such choices instead).

In what follows, we first review the cut-offs model, before describing the empirical context of the choice experiment being analysed here. The design of the choice experiment is then reviewed. Results are followed by a discussion.

\section{The "cut-offs" model in Choice Experiments.}

In this paper, we make use of the "cut-offs" choice experiment approach proposed by Swait (2001). As originally set out, this attempts to deal with the limited ability of standard choice modelling approaches to represent non-compensatory preferences. Non-compensatory preferences imply that consumers can no longer be assumed to have smooth, continuous indifference curves, such that any change in environmental quality can be compensated for by a finite change in a numeraire good such as income. Here, we extend the approach to deal with choice inconsistency.

In choice modelling, we typically assume respondents to be rational individuals who maximise their utility by choosing alternatives from a finite choice set that brings them the highest utility. Following the theoretical framework of Swait (2001), a typical formulation of the choice problem is: 
[Max] $U=\sum_{i \in C} \delta_{i} U\left(X_{i}\right)$

s.t. $\quad \sum_{i \in C} \delta_{i}=1 ; \quad \sum_{i \in C} \delta_{i} p_{i} \leq Y ; \quad \delta \in\{0,1\} \quad \forall i \in C$.

where $\mathrm{U}$ is the utility, $\mathrm{C}$ is the set of substitute alternatives such as alternative nature-based tourism experiences, $\delta_{\mathrm{i}}$ is a choice indicator equal to 1 if respondents choose alternative $i$ and 0 otherwise, $p_{i}$ is the price of alternative $i, \mathrm{X}_{\mathrm{i}}$ is the $k$ dimensional vector that describes the good, and $\mathrm{Y}$ is respondents' income.

In this context, respondent $n$ is typically assumed to consistently evaluate all the attribute tradeoffs between competing alternatives. However, many other decision rules may be used by respondents, depending on factors such as the difficulty of the choice task, their knowledge about the goods under study, and the environmental and social conditions in which the choice is carried out. Cut-offs can be viewed as a non-compensatory choice heuristic thought to simplify choices in a world of costly decision-making. The use of cut-offs suggests that individuals are unwilling or unable to maximise utility by considering all possible choices and their pay-offs, relative to their budget constraint (Svenson, 1996). Here, though, we take a different interpretation: we assume that stated cut-offs represent limits to acceptable trade-offs between the attributes of goods.

Swait (2001) notes that such cut-offs may be thought of as "hard" or "soft". Hard cutoffs are attribute levels that must be reached before a choice is allowed. Including hard cutoffs into the choice modelling framework requires adding additional constraints that prevent respondents from choosing an alternative that violates any of their stated cut-offs. For example, if respondent $n$ stated that he would not pay more than $x^{1}$ for a good (the hard cut- 
offs value), the utility maximization process only considers all the alternatives with a cost less than $x^{1}$. Equation (1) is then rewritten as:

[Max] $U=\sum_{i \in C} \delta_{i} U\left(X_{i}\right)$

s.t. $\quad \sum_{i \in C} \delta_{i}=1 ; \quad \sum_{i \in C} \delta_{i} p_{i} \leq Y ; \quad \delta \in\{0,1\} \quad \forall i \in C ;$

$\delta_{i} \theta^{L} \leq \delta_{i} Z_{i} ; \delta_{i} \theta^{U} \geq \delta_{i} Z_{i} ; \quad \delta \in\{0,1\} \quad \forall i \in C$.

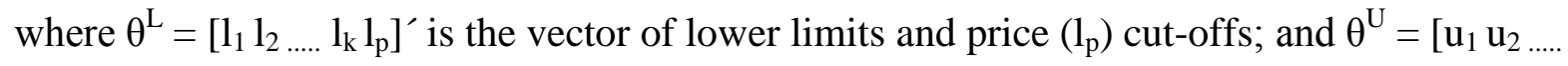
$\left.\mathrm{u}_{\mathrm{k}} \mathrm{u}_{\mathrm{p}}\right]^{\prime}$ is the vector of upper limits and price $\left(\mathrm{u}_{\mathrm{p}}\right)$ cut-offs and $\mathrm{Z}_{\mathrm{i}}$ is a $k+1$ dimensional vector that describes alternative $i\left(\mathrm{X}_{\mathrm{ik}}\right)$ where the additional dimension is the price (i.e., $\left.\mathrm{Z}_{\mathrm{i}(\mathrm{k}+1)}=\mathrm{p}_{\mathrm{i}}\right)$.

However, cut-offs need not be hard: consumers can choose to violate them if the benefits are great enough (that is, once the opportunity costs of self-imposed hard cut-offs are recognised). This approach was first proposed by Huber and Klein (1991). Soft cut-offs can be used to represent non-linearities or kinks in the deterministic portion of the utility function. Swait (2001) claimed, and showed for his data on rental car choices, that use of a soft cut-offs model would provide a better fit to stated choice data; this was also found by Amaya-Amaya and Ryan (2006) for two stated choice data sets for health care options. Swait also notes that ignoring the presence of soft cut-offs where these are in fact present in peoples' decision making will lead to biased estimates of marginal utilities.

Making the cut-offs "soft" requires adding to the utility function a penalty function associated with cutoff violations:

\footnotetext{
${ }^{1}$ The same would apply in case of a lower limit cut-offs, for instance if respondent declares he/she would not select any alternative cheaper than $x$.
} 
[Max] $U=\sum_{i \in C} \delta_{i} U\left(X_{i}\right)+\sum_{i \in C} \sum_{k} \delta_{i}\left(w_{k} \lambda_{i k}+v_{k} \kappa_{i k}\right)$

s.t. $\quad \sum_{i \in C} \delta_{i}=1 ; \quad \sum_{i \in C} \delta_{i} p_{i} \leq Y ; \quad \delta \in\{0,1\} \quad \forall i \in C ;$

$\delta_{i}\left(\theta^{L}-Z_{i}\right)-\lambda_{i} \leq 0 \quad \delta \in\{0,1\} \quad \forall i \in C ;$

$\delta_{i}\left(Z_{i}-\theta^{U}\right)-\kappa_{i} \leq 0 \quad \delta \in\{0,1\} \quad \forall i \in C ;$

$\lambda_{i} \geq 0 ; \kappa_{i} \geq 0 ; \quad \forall i \in C$.

where $w_{k}$ is the marginal disutility of violating the lower cutoff for attribute $k(\mathrm{k}=1 \ldots \mathrm{K}+1) ; v_{k}$ is the marginal disutility of violating the upper cutoff for attribute $k(\mathrm{k}=1 \ldots \mathrm{K}+1)$; $\lambda_{\mathrm{ik}}$ is a cutoff constraint variable for the lower limit cut-offs and $\kappa_{\mathrm{ik}}$ is a cutoff constraint variable for the upper limit cut-offs. The coding of such cut-offs constraints is straightforward. For quantitative attributes $\lambda_{\mathrm{ik}}=\max \left(0, \theta^{\mathrm{L}}{ }_{\mathrm{k}}-\mathrm{Z}_{\mathrm{ik}}\right), \kappa_{\mathrm{ik}}=\max \left(0, \mathrm{Z}_{\mathrm{ik}}-\theta^{\mathrm{U}}{ }_{\mathrm{k}}\right)$ where $(\mathrm{k}=1 \ldots \mathrm{K}+1)$; for qualitative attributes $\lambda_{\mathrm{ik}}$ and $\kappa_{\mathrm{ik}}$ are equal to 0 or 1 depending if the stated cut-offs have been violated or not. Note that if a choice alternative satisfies all cut-offs, the optimal solution has all $\lambda_{\mathrm{ik}}$ and $\kappa_{\mathrm{ik}}$ equal to zero, thus the utility maximization problems reduces to equation (1).

In this model specification, we use a linear utility function in which the marginal effects of each attribute on utility will be affected by the disutility of cut-offs violation. In particular, we assume:

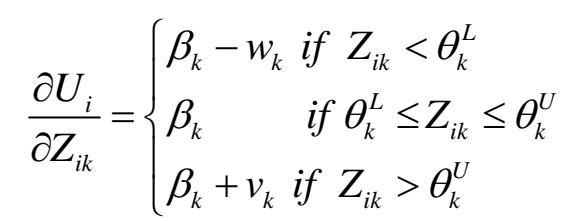

The suggestion made in this paper is that attribute cut-offs can also be used as a way to identify choices which are inconsistent with what appears to be respondents' maximum WTP for the good in question. We can test for such behaviour in a simple way by specifying hard cut-offs constraints when respondents violate their upper price stated cut-offs - the most they 
say they are willing to pay for any specification of the good within the attribute space defined in the choice experiment - by more than an exogenously specified value. For instance, if respondent $n$ declared that he/she would not be willing to pay more than $100 €$ for a whalewatching trip, the "soft" cut-offs approach allows him/her to pay more (say $120 €$ ) when the alternative offers her some compensating features that give her greater benefits than the marginal cost above the cutoff, albeit with a utility penalty for violating the soft cut-off. However, this can viewed as acceptable only up to a specific degree of cutoff violation. A constraint can then be added to the maximization problem by requiring that the cut-offs violations for price cannot be greater than a percentage of the respondents' stated maximum WTP (their cutoff value for the price attribute);

[Max] $U=\sum_{i \in C} \delta_{i} U\left(X_{i}\right)+\sum_{i \in C} \sum_{k} \delta_{i}\left(w_{k} \lambda_{i k}+v_{k} \kappa_{i k}\right)$

s.t. $\quad \sum_{i \in C} \delta_{i}=1 ; \quad \sum_{i \in C} \delta_{i} p_{i} \leq Y ; \quad \delta \in\{0,1\} \quad \forall i \in C ;$

$\delta_{i}\left(\theta^{L}-Z_{i}\right)-\lambda_{i} \leq 0 \quad \delta \in\{0,1\} \quad \forall i \in C ;$

$\delta_{i}\left(Z_{i}-\theta^{U}\right)-\kappa_{i} \leq 0 \quad \delta \in\{0,1\} \quad \forall i \in C ;$

$\delta_{i} \kappa_{i p} \leq \gamma \theta_{p}^{U} \quad \forall i \in C \quad \gamma \in R^{+}$

$\lambda_{i} \geq 0 ; \kappa_{i} \geq 0 \quad \forall i \in C ;$

where $\gamma$ is an exogenous value set by the analyst. This value represents the amount of the violation (as a percentage relative to upper price cutoff) that the analyst is willing to accept. To respondents whose preferred alternatives cost more than the upper price cut-offs (maximum WTP) but which are lower than the value of $\gamma$, then the "soft" cut-offs approach will be applied. Choices whose ratio $\kappa_{\mathrm{ip}} / \theta_{\mathrm{p}}^{\mathrm{U}}$ is greater than the $\gamma$ value (i.e., the price violation is too large with respect to what can be considered "acceptable") are then treated as 
if they chose the zero-cost opt-out option ${ }^{2}$. In the case of nature-based tourism, for example if a respondent declares that he is willing to pay as maximum $100 €$ to take a trip and later chooses an alternative that costs $200 €$, there are clues to think that his choice is inconsistent. If the analyst is willing to accept a violation of the upper price cut-offs of $50 \%$ as a maximum (i.e. $150 €$ ) this respondent would be treated as they had chosen the "no trip" choice.

Which value to use as an acceptable limit is an empirical question that the analyst has to address by undertaking a sensitivity analysis using different " $\gamma$ values". In this study we test several " $\gamma$ values" extending over the interval [0.04-1.5]: results are reported in section 5 . Inconsistent choices, defined in this way, could also be simply deleted from the data set, rather than being re-coded as "no trip": results are also reported for this treatment.

\section{Case Study: Gorilla Tourism in Rwanda.}

In many developing countries, tourism is providing an increasingly important source of export earnings and foreign direct investment (Wunder, 2000). In Rwanda, nature tourism is a particularly dynamic sub-sector, thanks to the charismatic mountain gorilla population found in the Volcanoes National Park (VNP) in the north-west of the country. VNP consists of about $160 \mathrm{~km}^{2}$ of montane forest and until Rwanda's independence in 1962 was part of Africa's first national park, the Parc National Albert, created in 1925 with an intention of protecting the great apes (ORTPN, 2004). Both the mountain gorillas and the VNP as a tourist destination became internationally renowned through the work of the conservationist Dian Fossey who died in 1986 and whose biography was later turned into the popular movie “Gorillas in the Mist”. By the early 1980's Rwanda was receiving up to 22,000 visits to the national parks annually. However, visits collapsed during the genocide, civil war and

\footnotetext{
${ }^{2}$ As Swait (2001) pointed out, the set $\mathrm{C}$ must have a null alternative (i.e. the possibility of not choosing), otherwise the utility maximization problem might not have a feasible solution for particular configuration of attributes and cut-offs.
} 
subsequent period of insecurity from 1994 to 1998 (ORTPN, 2004). Despite recent threats to the gorillas from illegal hunting, today the park is well protected, and numbers of the great apes are increasing (Gray et al, 2005). Since the park was re-opened in 1999 its tourism industry has seen an incredible rebound from 417 park visits in that year to around 30,000 park visits in 2006.

The Virunga mountain gorilla (Gorilla beringei beringei) is a highly endangered African ape subspecies, with a total estimated population of 380 existing only in the Virunga Conservation Area encompassing Rwanda, Democratic Republic of Congo and Uganda (Homsey, 1999; Fawcett et al, 2004) The distribution of the Virunga mountain gorillas is limited to an approximate area of $447 \mathrm{~km}^{2}$, which encompasses the Mgahinga Gorilla National Park in Uganda, the Parc National des Volcans of Rwanda and the Mikeno sector of the Parc National des Virunga of the Democratic Republic of Congo. The current population size of 380 individuals represents a $17 \%$ increase from 1989, when a complete census estimated 324 individuals. The Virunga mountain gorilla represents an isolated island population in an upland area surrounded by a sea of humanity at some of the highest human densities found on the African continent (some areas reach 820 people per $\mathrm{km}^{2}$ ) with extremely poor, agricultural-based local economies (Plumptre et al, 2004). Gorillas are and will continue to be severely threatened by anthropogenic disturbance, such as agricultural conversion and illegal extraction of resources.

Nature-based or eco-tourism is an approach to promoting both environmental and social development goals. The client base for gorilla tourism in Rwanda is broad, the gorillas being visited by independent traveller, over-landers and high-end tours (ORTPN, 2004). Tourism development strategies based on market differentiation are being developed regionally. Distinct pressures exist on national parks authorities to maximize their revenues in order to be able to finance their conservation and tourism activities. There is thus a critical 
balance to be achieved between exploitation of theses resources through tourism, and their conservation. To protect gorilla health and behaviour, limits have been agreed within the VNP regarding the number of groups "habituated" for tourism as well as the number and duration of tourist visits. This limit currently stands at a maximum of 8 tourists, for one hour, once per day with each habituated gorilla group designated for tourism (Homesy, 1999). Moreover, recent work has shown that current conservation measures impose costs on local communities, in terms of restrictions in access to forest areas and restrictions on hunting and food and fuel collection (Bush, 2008). This means that future measures which can at least partly compensate local people for these costs would be particularly valuable. For this reason, we include the percentage of tourism revenues returned to local people as one of the attributes in the choice experiment design described below.

\section{Study Design}

To aid questionnaire design, focus groups and pilot interviews were conducted in June and July 2005 with groups of visiting tourists in Volcanoes National Park (VNP), to identify the key attributes that visitors to the gorillas were concerned about. Collection of the main survey data ran from August 2005 until January 2006. In total 426 surveys were administered, of which 419 were returned complete and useable. Respondents were identified at random each morning when they arrived for gorilla trekking at the VNP and asked if they would participate later that day in the survey. They were later approached in their accommodation, in and around Ruhengeri Town and Kinigi Village to fill out the questionnaires on 1) personal socioeconomic and demographic characteristics, related tourism activities and interests, 2) the choice task (with nine sets/cards per respondent) and 3) the cut offs. 
A total of 18 different choice sets were developed following the design procedure proposed by Street et al $(2005)^{3}$, which were separated into two different blocks. Following extensive focus group discussions, the choice attributes included in the design were trek group size, length of trek, the possibility of seeing other wildlife, community benefits from tourism and a price parameter representing possible future increases in current trekking fees (see Table 1 and Figure 1). The current trek price at the time of the survey was $\$ 375$, and almost all treks were fully-booked, indicating that it was likely that some people would have been willing to pay more for the experience. Prices have since risen to $\$ 500$ per trip, and treks are still fullybooked.

We experimented with cut offs presented both before and after the choice experiment in order to assess the impact of cut-off questions on the completion of the choice task ${ }^{4}$. To accomplish this, we split the sample into two halves; to the first we asked for the cut-offs before the choice task. The second group were asked to provide information on their cut-off levels after the choice questions. Cut offs were identified for maximum trek group size, the minimum and maximum length of trek, the minimum $\%$ of tourism receipts being channelled to the local community, and maximum willingness to pay over current permit price specified by each respondent (see Table 2), leading to a total of 6 cut-off parameters to be estimated ${ }^{5}$.

\footnotetext{
${ }^{3}$ The design efficiency was $94.07 \%$ efficient and main effects were perfectly uncorrelated. The design was attribute balanced and does not contain any dominated choice sets.

${ }^{4}$ Swait (2001) commented that the positioning of cut-off questions was an interesting avenue for future work.

${ }^{5}$ For example for the price cutoff, we asked: "What is the maximum additional amount above the current permit price that you would be willing to pay?"
} 


\section{Econometric Approach}

We used a Random Paremeters Logit model to analyse the choice data (for details on this approach, and how it compares with multi-nomial logit, see Henscher et al, 2005). In the standard random utility framework, implementing a cut-offs model modifies the deterministic part of the utility function to incorporate a penalty when soft cut-offs have been violated. The size of cut-off violation and their associated utility penalties $\lambda_{\mathrm{ik}}$ and $\kappa_{\mathrm{ik}}$ with their coefficients $w_{k}$ and $v_{k}$ are added to the deterministic component of utility. Considering a linear-in-utility function ( $V_{i n}=\sum_{k} \beta_{k} X_{i n k}$ ) defined over attributes $X$, the deterministic part of utility becomes

$$
V_{i n}=\sum_{k}\left(\beta_{k} X_{i n k}+w_{k} \lambda_{i n k}+v_{k} \kappa_{i n k}\right)
$$

In the random parameter modelling framework, the utility attached to each attribute is allowed to vary over individuals:

$$
V_{i n}=\sum_{k}\left(\beta_{k} X_{i n k}+\eta_{\mathrm{kn}} X_{i n k}+w_{k} \lambda_{i n k}+v_{k} \kappa_{i n k}\right)
$$

where $\eta_{k \mathrm{n}}$ is a vector of $k$ deviation parameters which represents an individual's tastes relative to the sample average $(\beta),\left(\beta_{\mathrm{kn}}=\beta_{\mathrm{k}}+\eta_{\mathrm{kn}}\right)$. The $\eta$ terms, as they represent personal tastes, are assumed constant for a given individual across all the choices they make, but not constant across people. Random parameter logit probabilities are weighted averages of the logit formula evaluated at different values of $\beta$, with the weights given by the density $f(\beta)$. The probability that respondent $n$ chooses alternative $i$ is given by:

$$
P_{n i}=\int L_{n i}(\beta) f(\beta) d(\beta)
$$


where $\mathrm{L}_{\mathrm{ni}}(\beta)$ is the logit probabilities evaluated at parameters $\beta$. Since this integral has no closed form, parameters are estimated through simulation and maximising the simulated loglikelihood function. In order to estimate the model it is necessary to make an assumption over how the $\beta$ coefficients are distributed over the population. Here we assume that preferences for all the attributes follow a normal distribution, based on the results of focus groups and pretesting. This means, for example, that we allow some people to prefer longer treks and some to prefer shorter treks. The price parameter was initially allowed to vary across respondents as well. However, testing showed that heterogeneity in preferences towards price disappeared once we use the stated upper price cut-offs to edit choices. Thus, the reported models show the price term as non-random.

The criteria used in selecting a value for $\gamma$ - in equation (5), the exogenous censoring parameter for choices - included model fitting and degree of implied censoring of choices. Since larger values of the $\gamma$ parameter allows for larger violations of the price cutoff to be counted in the choice model as a purchase decision, there is an argument for not using values of $\gamma$ that are "too large", if the intention is to mitigate against the tendency of respondents to give price-inconsistent responses. On the other hand, smaller values of $\gamma$ imply an increasingly-strict censoring of choices, in that the number of choices re-classified will be a decreasing function of the value of $\gamma$. In terms of model fitting, Figure 2 shows that the maximum likelihood of the model at convergence is pretty stable at small values of the $\gamma$ parameter. It decreases gently until it reaches the $\gamma$ value of 0.37 where it decreases sharply. The same could be said for the intervals of $\gamma$ values between 0.5 and 0.75 . Results revealed that when using low values of the $\gamma$ parameter (between 0.04 and 0.25 ) there is a significant increase of model fitting. When higher inconsistencies are allowed $(\gamma>1.5$ for instance) the model fitting decreases until it corresponds to that of the model with all inconsistencies. Sensitivity analysis showed that models estimated using a $\gamma$ values $=0.37$ are very similar to 
the model estimates using a $\gamma$ value of 0.75 . For example, setting $\gamma=0.50$ produces a parameter estimate for price equal to -0.002 and for its cut-off equal to -0.023 (both significant), values which are almost identical to those for $\gamma=0.75$, Consideration of both criteria (model fitting and degree of censoring of choices) led us to choose a value of $\gamma=0.75$ (75\%) for reporting of choice model results: estimates for other values of $\gamma$ can be obtained from the authors.

\section{Results}

Table 2 shows the cut-off values stated by respondents. Looking at the price attribute, $33 \%$ of respondents said that they were not willing to pay any increase in the price of a trek, whilst the mean maximum WTP above current price was $\$ 95$. Table 3 shows detail on cut-off violations in terms of the actual choices made by the respondents, compared to their individually-stated cut-offs. The key fact that emerges from Table 3 is that many choices involve the violation of stated cut-offs: as noted in section 2, this is permissible in the context of the model, although violations will incur a utility penalty. The greatest number of violations occurred for the price and the community benefit attributes, with just under half of respondents choosing options which violated their stated cut-offs. Violations were lowest for tour group size. A statistical test showed that there were no significant differences in the frequency of cut-offs violation comparing the sub-samples where the cut-offs are elicited before or after the choice task $\left(\chi^{2}{ }_{4}=4.45\right.$, sig. 0.34$)$. Furthermore, a likelihood ratio test of the null hypothesis that the estimated preference parameters are independent of the positioning of the cut-offs question (before of after the choice tasks) fails to reject this null.

Econometric results are organised as follows. First, we present a model estimated on all data but without the cutoff terms, and then compare this to a model which includes soft cut-offs. Secondly, we consider the impacts of reclassifying choices where people violate 
their stated maximum WTP (price cutoff) by at least 75\% (see below for how this value for $\gamma$ was chosen) as "no trip" choices. Third, we repeat the exercise for people violating their stated maximum WTP by at least $75 \%$, but this time deleting these from the dataset before the choice model is estimated, rather than re-classifying them as "no trip".

\section{$\underline{6.1 \text { Using all the choice data: soft cut-offs versus no cut-offs }}$}

This is the comparison closest to the Swait (2001) paper. Table 4 shows the no cut-offs results compared to the results with soft cut-offs. Note that the model fitting improves between columns 1 and 2 (this improvement is significant at 99\%). However, the price attribute has a positive parameter in both cases, whilst the parameter on the upper price cutoff is not statistically significant. The positive sign on the price variable might be explained by respondents considering that price reflected some other desirable attribute of a gorilla trek that was not described by the other attributes (which cannot be tested for), or by some respondents not paying attention to the price attribute. We also note that all individuals chose either A or B in the choice sets answered - that is, none chose the "no trip" option. This is not surprising when one considers that, for most individuals, the main point of their (expensive) visit to Rwanda is to go on a gorilla trek. However, choice inconsistencies might lie behind the positive sign on price result. To investigate this we now re-code trip choices that violate respondents" stated maximum WTP by $75 \%$ or more as "no trip".

\section{$\underline{6.2}$ Re-classifying inconsistent responses as "no trip".}

Setting $\gamma$ such that any choice which violates a person's stated maximum WTP in terms of its price by at least $75 \%$ is reclassified as a stay-at-home choice produces big effects. 
Model fitting improves significantly both with and without the inclusion of cut-off penalties in the choice model (Table 5). The parameter on price becomes negative in the no cut-offs version (suggesting that it was trip choices by those inconsistent respondents who violated their maximum stated WTP which are responsible for the positive price effect), whilst in the cut-offs version the cutoff parameter on price is also now significant, and 10 times bigger than the price coefficient. This implies a steep kink in the marginal disutility of higher prices above the upper soft cutoff. Adding soft cut-offs to this edited data set of choices produces, in itself, a significant improvement in the model's explanatory power: compare, for example, the $t$ statistics on the random parameters in the utility function. It may also be seen that, whereas the parameter estimate on a "high" chance of seeing other wildlife on the trek is insignificant in Table 4, it becomes significant (at the 90\% level) and positive once inconsistent responses - here, those where maximum WTP is exceeded by at least $75 \%$ - are removed.

Looking at the parameter estimates for the non-price attributes, it may be seen that visitors prefer smaller tour groups; prefer a length of trek between 1 and 3 hours to either shorter or longer treks; and prefer to see greater numbers of other wildlife in addition to gorillas. However, there is no evidence of a significant effect for what percentage of park revenues are recycled to local communities in the national park: the parameter in $C B$ is insignificantly different from zero at the $95 \%$ significance level. In terms of preference heterogeneity, we find significant evidence of this for tour group size, seeing other wildlife (partially), and community benefits. This can be seen by observing the statistically significant standard deviation parameters in Table 5. However, the parameter estimates for the standard deviation terms (differences in values held by individuals relative to the mean value) for the attributes relating to length of trek and the highest level of other wildlife viewing opportunities are not significant at even the $90 \%$ level. 


\subsection{Deleting inconsistent choices}

An alternative editing rule for choices which violate the price cutoff (stated maximum WTP) by at least $75 \%$ is to delete them from the dataset, rather than re-classifying them as "no trip" choices. This yields the results shown in Table 6 . In the no cut-offs version, the price parameter remains as negative and highly significant. However, when cutoff parameters are included, the coefficient on price becomes insignificantly different from zero, whilst the price cutoff parameter is negative and highly significant. This suggests that, once individuals who violate their cutoff by at least $75 \%$ are deleted from the data, then prices do not effect choices so long as they remain below an individual's cut-of value. But once choices are made which exceed maximum stated WTP, this comes at a penalty in terms of the deterministic component of utility.

\section{$\underline{6.4 \text { Implicit prices }}$}

The best fitting model is thus a model with soft cut-offs which re-classifies those choosing options which violate their stated upper price cutoff by at least $75 \%$ as "take no trip" choices (Table 5). Focussing these results, it is possible to examine the effects on implicit prices (marginal willingness to pay amounts) of including cut-offs in the choice model. There are four possible cases for defining implicit prices in such a model:

(1) no cut-offs are violated. The implicit price for an attribute such as tour group size (Tgs) is equal to (- $\beta$ tgs $/ \beta$ price).

(2) the cutoff for any attribute is violated, but not the cutoff for price. In this case, the implicit price for $\operatorname{tgs}=-(\beta \operatorname{tgs}+\beta$ cutoff tgs $) / \beta$ price

(3) the cutoff for price is violated but not the cut-offs for the other attributes. In this case, the implicit price for $\operatorname{tgs}=-(\beta$ tgs $) /(\beta$ price $+\beta$ cutoff price $)$ 
(4) both sets of cut-offs are violated: Implicit price $=-(\beta$ tgs $+\beta$ cutoff tgs $) /(\beta$ price + $\beta$ cut-offs price)

In Table 7, we present implicit prices for each of the attributes evaluated using (3) above, since the effect of violating the price cutoff turns out to be most important for this data and compare these to implicit prices evaluated assuming that no cut-offs are violated as in (1) above. Note that the validity of these implicit price estimates depends on how reasonable one believes the choice editing rule used in Table 5 to be. The effects on the implicit prices are indeed significant. For example, looking at tour group size, willingness to pay for a one person reduction in the number of people in the tour group falls from $£ 73$ in the no cut-offs version to $£ 18$ in the price cut-offs version. The same effect is found for all the other attributes. That is, mean WTP falls significantly once we take into account the soft cutoff penalty of the price of a trip, having re-classified choices that violate stated maximum WTP by more than $75 \%$ as "take no trip".

\section{Discussion and Conclusions}

This paper has proposed use of a cut-offs approach to choice modelling to address the issue of choice consistency, focussing very much on the cost attribute since this is key to welfare measurement. Choosing options which were more expensive than the most people said they would pay was found to result in an upward bias in implicit prices. We used violations of the stated price cut-off as a way of eliminating this bias, in the sense of detecting instances where individual choose an option which violates their stated maximum WTP by more than a particular amount, and penalising all maximum stated WTP choices using the cutoff penalty function. This echoes the practice of early open-ended contingent valuation studies, where large WTP values might be deleted as outliers, often in a rather arbitrary manner (for instance, through the use of $\mathrm{x} \%$ trimmed means). A cut-offs approach also allows 
us to represent a deterministic utility function which contains kinks around the cut-off levels. Swait (2001) states that since breaks in the utility function are person-specific, that "..fit improvements over models without (cut-offs) should be, and are, striking” (p914). We also find an improvement in model fitting by incorporating cut-offs; although not to the extent found by Swait. We find that only a minority of penalty function parameters are significant, but that the parameter on the penalty function for the price attribute was much bigger than that on the price attribute itself. This is evidence of marked non-linearities in demand.

Why would people make choices which violate their stated cut-offs? In the model presented in section 2, we argue that this is rational if the dis-utility from cut-off violation is more than compensated for by positive utility from the levels of other attributes in a choice alternative. However, we also noted that such behaviour may result from respondents not paying enough attention to the price ticket. The good we study here is somewhat distinctive in that for most people, their principal motivation for visiting Rwanda is to go on a gorilla trek. This is indeed a unique good, and one for which people are WTP considerably more than the current (high) price. Yet we also argued that the analyst should treat choice responses which violate the price cut-off by more than some "reasonable" limit value with caution. The approach taken here was to investigate what this reasonable limit might be in terms of maximising explanatory power of the choice model, whilst at the same time not imposing an overly-strict censoring rule on choices. Editing choices using this approach produced a model which might be judged as superior on a number of grounds.

Another reason why one might observe choices being made which violate stated cutoffs is that people are learning about their preferences as they progress through the survey tasks, and that asking them to re-consider their stated price cut-off after they have made a choice which violated it would be fruitful. Alternatively, people could be asked to re-consider their choices (both these suggestions are being followed up in a survey the authors are 
currently undertaking). However, if this preference learning explanation was correct then one would expect to see a difference in results from asking the cut-off questions before, as opposed to after, the choice tasks. This split-sample comparison was made here, and no significant difference found in responses in terms of parameter estimates. Preference learning would thus not be supported by the data in this instance as an explanation for choices which violate stated acceptable limits for individual attributes.

In conclusion, the cut-offs approach offers a useful way of imposing consistency on choices, whilst editing choices in this manner has an appreciable impact on estimated willingness to pay. The edited cut-offs model of choice proposed here would seem to offer some attractions to researchers in its ability to indentify inconsistent choices, as well as its well-known ability to allow for kinks in the utility function. The approach also gives analysts a new way of controlling for the coherence of stated choices, which might be particularly useful in internet and mail survey choice experiments where it is hard to de-brief respondents about their choices to make sure that they are internally consistent. However, issues remain with regard to explaining why people apparently violate their stated maximum willingness to pay. We also note that use of what is essentially an open-ended contingent valuation question to elicit peoples' maximum willingness to pay (their upper price cut-off) might be viewed as problematic, given the well-known problems with open-ended payment questions (Bateman et al, 2002). Whether the edited version of the cut-offs approach is successful in addressing the problem of hypothetical market bias in stated choices also remains to be tested. Finally, it would be interesting to explore the effects of using choice editing rules based on stated cutoffs for attributes other than price.

We thank ESRC for funding Bush's PhD, and the Dian Fossey Gorilla Fund International and the Great Ape Survival Fund, United States Agency for International Development for 
funding this work. We also thank participants at the 2008 EAERE conference and the 2008 Environmental Economics Hub Choice Modelling Workshop in Brisbane for comments, and two anonymous referees. 


\section{References}

Amaya-Amaya., M and Ryan, M (2006) "Incorporating attribute cut-offs in health care discrete choice models" Discussion paper, Health Economics Research Unit, Aberdeen.

Amir, O. and Levev, J. (2008) "Choice construction versus preference construction: the instability of preferences leaned in context” Journal of Marketing Research, 45 (2), 145-158.

Bateman, I. et al (2002) Economic Valuation with Stated Preference Techniques. Cheltenham: Edward Elgar.

Campbell, D. (2008) "Identification and analysis of discontinuous preferences in discrete choice experiments" Paper to EAERE conference, Goteborg, June.

Carlsson F and Martinsson P (2001) "Do hypothetical and actual willingness to pay differ in choice experiments?" Journal of Environmental Economics and Management, 41, 179-192.

DeShazo J.R. and Fermo G. (2002) "Designing choice sets for stated preference methods: the effects of complexity on choice consistency" Journal of Environmental Economics and Management, $44(1), 123-143$.

Fawcett K, Hodgkinson, C \& Mehlman P 2004. “An assessment of the impact of tourism on the Virunga mountain gorillas' Technical report, Diane Fossey Gorilla Fund International, Atlanta, USA.

Gray, M., McNeilage, A., Fawcett, K., Robbins, M. M., Ssebide, B., Mbula, D. and Uwingeli, P. 2005. "Virunga Volcanoes range and census, 2003.” Joint organizers report. Uganda Wildlife Authority/Office Rwandaise du Tourisme et des Parcs Nationaux/Institut Congolaise pour le Conservation du Nature.

Hanley N, Wright R.E. and Koop G. (2002) "Modelling recreation demand using choice experiments: rock climbing in Scotland" Environmental and Resource Economics, 22, 449-466. 
Harrison G. and Rustrom E. (2005) "Experimental evidence on the existence of hypothetical bias in value elicitation methods" in C.R.Plott and V.L.Smith (eds) Handbook of Experimental Economics. Amsterdam: North Holland.

Hensher, D., Rose, J. and Greene, W (2005) Applied choice analysis; a primer. Cambridge University Press, UK.

Homesy, J 1999. “Ape tourism and human diseases; how close should we get?” Technical Report for the International Gorilla Conservation Program. Nairobi, Kenya.

Huber J. and Klein N. (1991) “Adapting cut-offs to the choice environment: the effects of attribute correlation and reliability" Journal of Consumer Research, 18, 346-357.

List J., Sinha P., and Taylor M. (2006) "Using choice experiments to value non-market goods and services: evidence from field experiments" Advances in Economic Analysis and Policy, 6 (2), $1-37$.

ORTPN (2004) Strategic Plan 2004-2008. Draft version 2. Office Rwandais du Tourisme et des Parcs Nationaux, Kigali.

Plumptre, A.J., Kayitare, A., Rainer, H., Gray, M., Munanura, I., Barakabuye, N., Asuma, S., Sivha, M., and Namara, A. (2004) The Socio-economic Status of People Living Near Protected Areas in the Central Albertine Rift. Albertine Rift Technical Reports, 4.

Street, D., L., Burgess, and J. Louviere. (2005) 'Quick and easy choice sets: Constructing optimal and nearly optimal stated choice experiments.' International Journal of Research in Marketing, vol. 22, 459-470.

Svenson O., (1996) "Decion making and the search for fundamental psychological regularities" Organisational Behaviour and Human Decision Processes. 65 (3), 252-267.

Swait J. (2001) “A non-compensatory choice model incorporating attribute cut-offs" Transportation Research B, 35, 903-928. 
Wunder, S. 2000. Ecotourism and economic incentives-an empirical approach. Ecological Economics, 32, 465-479 


\section{Figure 1 Example choice card with pre-amble text.}

"We now wish you to review the choice cards below, there is no right or wrong answer we are simply interested in your opinion. For each of the 9 select which option would be most preferable to you:"

Choice 1

\begin{tabular}{|lccc|}
\hline Gorilla Trip Features & Option A & Option B & Neither \\
\hline $\begin{array}{l}\text { Tour group size } \\
\text { Length of trek }\end{array}$ & $\begin{array}{c}6 \\
\text { More than } 3 \\
\text { hours } \\
10 \% \text { Of } \\
\text { Community benefit }\end{array}$ & $\begin{array}{c}\text { Less than } 1 \\
\text { hour } \\
\text { 20\% Of permit } \\
\text { price }\end{array}$ & $\begin{array}{c}\text { I would not } \\
\text { choose either } \\
\text { option to visit } \\
\text { the gorillas } \\
\text { and would } \\
\text { conduct some } \\
\text { other activity } \\
\text { instead. }\end{array}$ \\
$\begin{array}{l}\text { Possibility of seeing } \\
\text { other wildlife } \\
\text { Increase in permit price }\end{array}$ & $\$ 75$ & $\$ 50$ & $\square$ \\
\hline $\begin{array}{l}\text { Which option do you } \\
\text { choose } \\
(\checkmark \text { one only) }\end{array}$ & $\square$ & $\square$ & $\square$ \\
\hline
\end{tabular}


Figure 2: Maximum log likelihood of different models estimated using different $\gamma$ values.

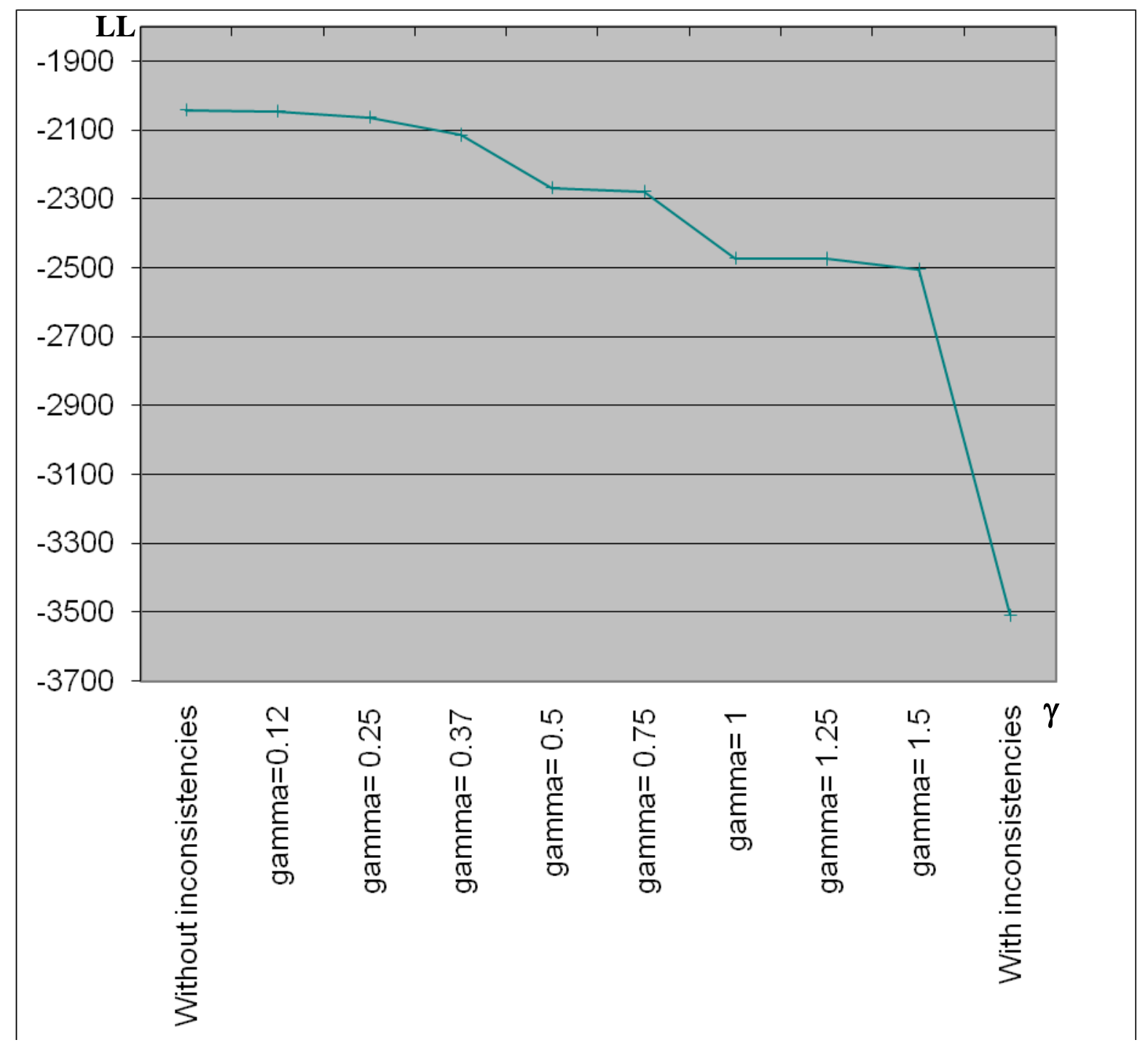


Table 1 Attributes and their levels

\begin{tabular}{|c|c|c|}
\hline Attribute & $\begin{array}{c}\text { Description (a more comprehensive } \\
\text { description was provided in the survey } \\
\text { instrument) }\end{array}$ & Levels \\
\hline Tour Group Size & $\begin{array}{l}\text { The number of tourists in a group. Limited to a } \\
\text { maximum of } 8 \text { for conservation reasons }\end{array}$ & $\begin{array}{l}\text { Small-4 } \\
\text { Medium-6 } \\
\text { Large-8 }\end{array}$ \\
\hline Length of trek & The amount of time taken to reach the gorillas. & $\begin{array}{l}\text { Short, <1hour } \\
\text { Medium, }>1 \text { but }<3 \text { hours } \\
\text { Long, }>3 \text { hours }\end{array}$ \\
\hline $\begin{array}{l}\text { Community } \\
\text { Benefit }\end{array}$ & $\begin{array}{l}\text { Currently } 20 \% \text { of gate gross park revenues is } \\
\text { diverted towards financing development } \\
\text { activities in communities adjacent to the national } \\
\text { park. Focus groups showed that some visitors } \\
\text { felt it is important that local communities receive } \\
\text { greater benefits from tourist spending. }\end{array}$ & $\begin{array}{l}\text { No change } \\
10 \% \text { more } \\
20 \% \text { more } \\
30 \% \text { more }\end{array}$ \\
\hline Other wildlife & $\begin{array}{l}\text { The ability of tourists to see other flora and fauna } \\
\text { the park can contribute to the richness of the trek } \\
\text { experience. }\end{array}$ & $\begin{array}{l}\text { High } \\
\text { Medium } \\
\text { Low }\end{array}$ \\
\hline $\begin{array}{l}\text { Permit price } \\
\text { increase }\end{array}$ & $\begin{array}{l}\text { Price increase on gorilla trek permit and implied } \\
\text { new total (including park entry fee) - figure in } \\
\text { parentheses shows new total fee. }\end{array}$ & $\begin{array}{ll}\$ 25 & (\$ 400) \\
\$ 50 & (\$ 425) \\
\$ 75 & (\$ 450) \\
\$ 100 & (\$ 475) \\
\$ 150 & (\$ 525) \\
\$ 200 & (\$ 575)\end{array}$ \\
\hline
\end{tabular}


Table 2 Cut off frequencies for the sample

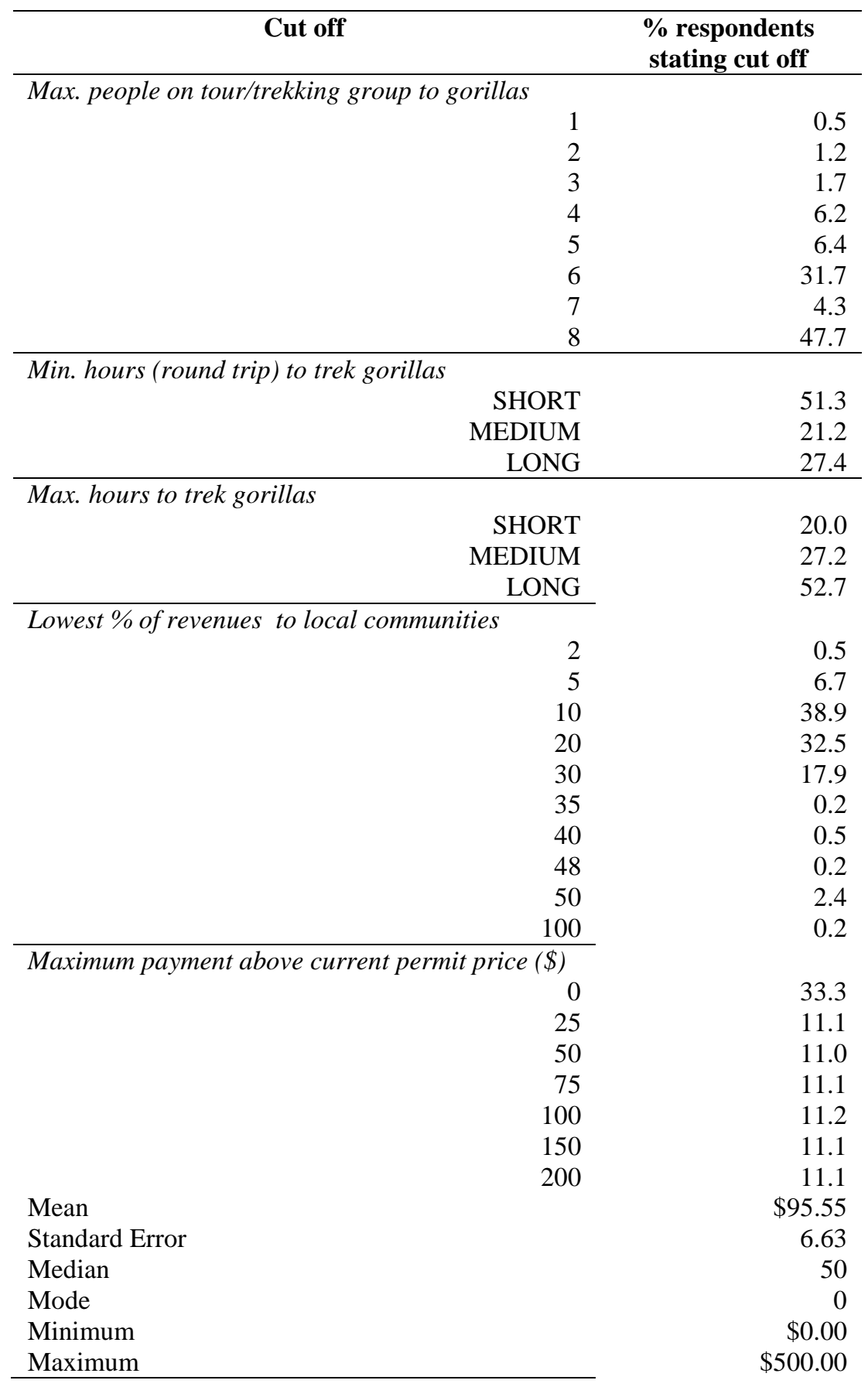


Table 3 Frequency of cut off violations

\begin{tabular}{|l|c|c|c|}
\hline & \multicolumn{3}{|c|}{$\begin{array}{c}\text { Number of people } \\
\text { violating their stated cut- } \\
\text { offs for any of their choices }\end{array}$} \\
\hline Versión & Before & After & Total \\
\hline Maximum length of trek & 47 & 35 & 82 \\
\hline Minimum length of trek & 40 & 39 & 79 \\
\hline Permit price & 87 & 107 & 194 \\
\hline Community benefit & 98 & 92 & 190 \\
\hline Tour group size & 41 & 35 & 76 \\
\hline
\end{tabular}


Table 4 Random Parameters Logit model all data, no cut-offs versus cut-offs.

\begin{tabular}{|c|c|c|c|c|}
\hline & \multicolumn{2}{|c|}{ No Cut-offs } & \multicolumn{2}{|c|}{ With soft cut-offs } \\
\hline & Parameter & T stat & Parameter & t-stat \\
\hline \multicolumn{5}{|c|}{ Random parameters in utility function } \\
\hline TGS & -0.129 & -5.66 & -0.133 & -5.51 \\
\hline LOT1 & 0.200 & 6.21 & 0.157 & 3.55 \\
\hline LOT2 & -0.13 & -0.41 & -0.139 & -3.10 \\
\hline $\mathrm{CB}$ & -0.007 & -2.62 & -0.004 & -0.84 \\
\hline OW1 & 0.197 & 6.12 & 0.192 & 5.92 \\
\hline OW2 & -0.31 & -0.99 & -0.023 & -0.74 \\
\hline \multicolumn{5}{|c|}{ Non-random parameters in utility function } \\
\hline Constant & 1.237 & 10.56 & 1.139 & 6.87 \\
\hline Price & 0.003 & 7.87 & 0.003 & 4.71 \\
\hline TG cutoff & & & 0.21 & 0.56 \\
\hline CB cutoff & & & -0.002 & -0.42 \\
\hline Price cutoff & & & 0.001 & 1.55 \\
\hline LOT cutoff 1 & & & -0.158 & -1.41 \\
\hline LOT cutoff 2 & & & 0.437 & 5.23 \\
\hline \multicolumn{5}{|c|}{ Standard deviations for parameter distributions } \\
\hline$\sigma \mathrm{TGS}$ & 0.34 & 19.30 & 0.341 & 19.24 \\
\hline$\sigma \mathrm{LOT} 1$ & 0.022 & 0.08 & 0.116 & 1.01 \\
\hline$\sigma \mathrm{LOT} 2$ & 0.172 & 2.17 & 0.091 & 0.62 \\
\hline$\sigma \mathrm{CB}$ & 0.000 & 0.03 & 0.002 & 0.11 \\
\hline$\sigma \mathrm{OW} 1$ & 0.001 & 0.01 & 0.000 & 0.00 \\
\hline$\sigma \mathrm{OW} 2$ & 0.012 & 0.15 & 0.006 & 0.07 \\
\hline Log Lik & -3524 & & -3506 & \\
\hline Pseudo $r 2$ & 0.14 & & 0.15 & \\
\hline$N$ (people, choices) & 419,3771 & & 419,3771 & \\
\hline
\end{tabular}

Notes: We used 100 replications and Halton draws.

TGS = total group size; LOT1 = length of trek between 1 and 3 hours (the reference is less than 1 hour); LOT2 = length of trek more than 3 hours; $\mathrm{CB}=$ community benefits OW $1=$ prob of seeing other wildlife $=$ medium (the reference is low); OW2 $=$ prob of seeing other wildlife : high.

The attributes TGS and price have upper cut-offs; CB has a lower cut-offs; LOT has both lower (LOT1) and Upper (LOT2) cut-offs. 
Table 5.

Choices violating upper price cut-off by $75 \%$ or more are re-classified as "take no trip"

\begin{tabular}{|c|c|c|c|c|}
\hline & \multicolumn{2}{|c|}{ No Cut-offs } & \multicolumn{2}{|c|}{ With soft cut-offs } \\
\hline & Parameter & T stat & Parameter & t-stat \\
\hline \multicolumn{5}{|c|}{ Random parameters in utility function } \\
\hline TGS & -0.626 & -13.325 & -0.423 & -9.604 \\
\hline LOT1 & 0.077 & 1.562 & 0.184 & 2.868 \\
\hline LOT2 & 0.035 & 0.730 & -0.111 & -1.756 \\
\hline $\mathrm{CB}$ & \begin{tabular}{|l|l|}
0.004 \\
\end{tabular} & 0.837 & 0.007 & 0.908 \\
\hline OW1 & 0.096 & 1.894 & 0.132 & 2.564 \\
\hline OW2 & 0.072 & 1.490 & 0.086 & 1.707 \\
\hline \multicolumn{5}{|c|}{ Non-random parameters in utility function } \\
\hline Constant & 1.481 & 8.817 & 0.881 & 3.701 \\
\hline Price & -0.009 & -11.233 & -0.002 & -1.992 \\
\hline TG cutoff & & & -0.012 & -0.236 \\
\hline CB cutoff & & & -0.006 & -0.732 \\
\hline Price cutoff & & & -0.022 & -12.689 \\
\hline LOT cutoff 1 & & & 0.047 & 0.310 \\
\hline LOT cutoff 2 & & & 0.395 & 3.430 \\
\hline \multicolumn{5}{|c|}{ Standard deviations for parameter distributions } \\
\hline$\sigma \mathrm{TGS}$ & 0.647 & 16.572 & 0.440 & 13.597 \\
\hline$\sigma \mathrm{LOT} 1$ & 0.221 & 2.613 & 0.151 & 1.374 \\
\hline$\sigma \mathrm{LOT} 2$ & 0.127 & 0.777 & 0.089 & 0.632 \\
\hline$\sigma \mathrm{CB}$ & 0.021 & 2.488 & 0.020 & 2.286 \\
\hline$\sigma \mathrm{OW} 1$ & 0.265 & 3.467 & 0.280 & 3.639 \\
\hline бOW2 & 0.079 & 0.814 & 0.106 & 1.059 \\
\hline $\log \operatorname{Lik}$ & -2383 & & -2277 & \\
\hline Pseudo $r 2$ & 0.42 & & 0.45 & \\
\hline$N$ (people, choices) & 419,3771 & & 419,3771 & \\
\hline
\end{tabular}

Notes: We used 100 replications and Halton draws and an RPL estimation.

TGS $=$ total group size; LOT1 = length of trek between 1 and 3 hours (the reference is less than 1 hour); LOT2 = length of trek more than 3 hours; $\mathrm{CB}=$ community benefits OW1 = prob of seeing other wildlife $=$ medium (the reference is low); OW2 $=$ prob of seeing other wildlife : high.

The attributes TGS and price have upper cut-offs; CB has a lower cut-offs; LOT has both lower (LOT1) and Upper (LOT2) cut-offs. 
Table 6: Choices violating upper price cut-off by $75 \%$ or more are deleted.

\begin{tabular}{|c|c|c|c|c|}
\hline & \multicolumn{2}{|c|}{ No Cut-offs } & \multicolumn{2}{|c|}{ With soft cut-offs } \\
\hline & Parameter & T stat & Parameter & t-stat \\
\hline \multicolumn{5}{|c|}{ Random parameters in utility function } \\
\hline TGS & -0.447 & -9.46 & -0.343 & -7.87 \\
\hline LOT1 & 0.185 & 3.67 & 0.227 & 3.51 \\
\hline LOT2 & 0.037 & 0.77 & -0.084 & -1.32 \\
\hline CB & -0.004 & -1.07 & 0.006 & 0.75 \\
\hline OW1 & 0.113 & 2.24 & 0.117 & 2.31 \\
\hline OW2 & -0.004 & -0.09 & 0.013 & 0.25 \\
\hline \multicolumn{5}{|c|}{ Non-random parameters in utility function } \\
\hline Constant & 2.103 & 11.44 & 1.56 & 6.18 \\
\hline Price & -0.0033 & -4.09 & 0.0005 & 0.56 \\
\hline TG cutoff & & & -0.0404 & -0.75 \\
\hline CB cutoff & & & -0.0112 & -1.18 \\
\hline Price cutoff & & & -0.0155 & -8.93 \\
\hline LOT cutoff 1 & & & 0.0161 & 0.104 \\
\hline LOT cutoff 2 & & & 0.4073 & 3.37 \\
\hline \multicolumn{5}{|c|}{ Standard deviations for parameter distributions } \\
\hline$\sigma \mathrm{TGS}$ & 0.605 & 15.21 & 0.485 & 13.56 \\
\hline$\sigma$ LOT1 & 0.201 & 1.89 & 0.141 & 0.84 \\
\hline бLOT2 & 0.040 & 0.20 & 0.002 & 0.02 \\
\hline$\sigma \mathrm{CB}$ & 0.007 & 0.66 & 0.007 & 0.46 \\
\hline$\sigma \mathrm{OW} 1$ & 0.207 & 1.74 & 0.202 & 1.84 \\
\hline$\sigma \mathrm{OW} 2$ & 0.034 & 0.18 & 0.089 & 0.52 \\
\hline $\log \operatorname{Lik}$ & -2439 & & -2083 & \\
\hline Pseudo r2 & 0.26 & & 0.28 & \\
\hline$N$ (people, choices) & 347,2278 & & 347,2278 & \\
\hline
\end{tabular}

Notes: We used 100 replications and Halton draws and an RPL estimation.

TGS = total group size; LOT1 = length of trek between 1 and 3 hours (the reference is less than 1 hour); LOT2 = length of trek more than 3 hours; $\mathrm{CB}=$ community benefits OW $1=$ prob of seeing other wildlife $=$ medium (the reference is low); OW2 $=$ prob of seeing other wildlife : high.

The attributes TGS and price have upper cut-offs; CB has a lower cut-offs; LOT has both lower (LOT1) and Upper (LOT2) cut-offs. 
Table 7 Implicit prices and 95\% confidence intervals (US \$ per person per trip)

\begin{tabular}{|lcc|}
\hline & $\begin{array}{c}\text { Implicit Price Model } \\
\text { (1) }\end{array}$ & $\begin{array}{c}\text { Implicit Price Model } \\
\text { (3) }\end{array}$ \\
Attributes & & \\
TGS & & \\
& -72.9 & -17.8 \\
LOT1 & $(-90.9 ;-59.4)$ & $(-22.7 ;-13.8)$ \\
& 22.1 & 10.8 \\
LOT2 & $(2.2 ; 43.2)$ & $(0.9 ; 22.8)$ \\
& 17.1 & -1.6 \\
CB & $(-1.7 ; 37.7)$ & $(-13.2 ; 9.9)$ \\
& 0.4 & 0.3 \\
OW1 & $(-0.6 ; 1.3)$ & $(-0.4 ; 1.0)$ \\
& 30.8 & 14.8 \\
OW2 & $(8.9 ; 50.9)$ & $(6.8 ; 22.9)$ \\
& 28.1 & 12.9 \\
& $(5.8 ; 48.8)$ & $(4.7 ; 20.6)$ \\
\hline
\end{tabular}

Model 1 corresponds to the model of table 4, panel B without cut-offs;

Model 3 corresponds to the model of table 4, panel B with soft cut-offs, where the implicit prices are estimated considering cut-offs violations on the trip price attribute alone. 